\title{
Geometric Superinductance Qubits: Controlling Phase Delocalization across a Single Josephson Junction
}

\author{
Matilda Peruzzo®, ${ }^{1, *}$ Farid Hassani, ${ }^{1}$ Gregory Szep $\odot,{ }^{2}$ Andrea Trioni, ${ }^{1}$ Elena Redchenko, ${ }^{1}$ \\ Martin Žemlička, ${ }^{1}$ and Johannes M. Fink $\oplus^{1, \dagger}$ \\ ${ }^{1}$ Institute of Science and Technology Austria, Klosterneuburg 3400, Austria \\ ${ }^{2}$ King's College London, London WC2R 2LS, United Kingdom
}

(Received 10 June 2021; accepted 8 October 2021; published 24 November 2021)

\begin{abstract}
There are two elementary superconducting qubit types that derive directly from the quantum harmonic oscillator. In one, the inductor is replaced by a nonlinear Josephson junction to realize the widely used charge qubits with a compact phase variable and a discrete charge wave function. In the other, the junction is added in parallel, which gives rise to an extended phase variable, continuous wave functions, and a rich energy-level structure due to the loop topology. While the corresponding rf superconducting quantum interference device Hamiltonian was introduced as a quadratic quasi-one-dimensional potential approximation to describe the fluxonium qubit implemented with long Josephson-junction arrays, in this work we implement it directly using a linear superinductor formed by a single uninterrupted aluminum wire. We present a large variety of qubits, all stemming from the same circuit but with drastically different characteristic energy scales. This includes flux and fluxonium qubits but also the recently introduced quasicharge qubit with strongly enhanced zero-point phase fluctuations and a heavily suppressed flux dispersion. The use of a geometric inductor results in high reproducibility of the inductive energy as guaranteed by top-down lithography — a key ingredient for intrinsically protected superconducting qubits.
\end{abstract}

DOI: 10.1103/PRXQuantum.2.040341

\section{INTRODUCTION}

Superconducting qubits are highly engineerable quantum systems that are at the forefront of quantum technology due to the offered strong interactions, which result in fast and precise control, but also due to the similarity to existing microchip fabrication and the available diversity of circuit designs [1-3]. Creative new ideas on how to encode, store, and control single quanta in electrical circuits paired with state-of-the-art fabrication have not only led to a big push in coherence times but also facilitated the observation of many new quantum physics phenomena $[4,5]$.

One example of this development is the fluxonium qubit [6], a large-inductance rf superconducting quantum interference device (rf-SQUID) characterized by strong anharmonicity and flux tunability. The ability to create such

\footnotetext{
*matilda.peruzzo@ist.ac.at

$\dagger$ jfink@ist.ac.at
}

Published by the American Physical Society under the terms of the Creative Commons Attribution 4.0 International license. Further distribution of this work must maintain attribution to the author(s) and the published article's title, journal citation, and DOI. qubits with a small transition frequency and charge-matrix element around the flux sweet spot has led to the energy relaxation time $T_{1}$ being greatly extended [7-9] but driving the qubit transition also becomes increasingly difficult for the same reason. Focusing instead on coherence, the qubit can be made flux insensitive by increasing the shunting inductance due to enhanced quantum phase fluctuations. This bears a resemblance to the transmon qubit, where increasing the shunting capacitance decreases the chargenoise sensitivity via enhanced quantum charge fluctuations [10]. This limit has recently been explored in Ref. [11] using a suspended Josephson-junction array where the lowest qubit transition varies by only $100 \mathrm{MHz}$ across a full flux period and leading to a theoretically predicted flux-limited coherence time $T_{2}$ on the order of hundreds of microseconds. This ultrahigh impedance limit is difficult to enter in practice, because it requires fabrication of a large inductor without creating a sizable capacitance that would inevitably lower the first transition frequency of the qubit. The superinductance limit has therefore been widely believed to be inaccessible with conventional inductors $[6,12]$, until very recently [13].

So far, superinductors have been realized by means of kinetic inductance, either using disordered materials with strong electron scattering [14] or by taking advantage of 
the inductance of a long chain of large Josephson junctions $[11,12,15]$. However, these techniques come with their own challenges. Josephson-junction arrays and granular aluminum fragment the superconducting wave function, which leaves the qubit potentially affected by charge noise $[16,17]$ or disorder due to static charges [18] that may also limit their applications in metrology [19]. Josephsonjunction arrays are additionally subjected to critical current noise, which adds fluctuations to the value of the inductance [2]. In the case of granular aluminum qubits, there is a difference in critical temperature between the inductor and the junction that may lead the junction to attract quasiparticles and can give rise to a higher loss rate [14]. In addition, a high degree of reproducibility of thin films and tunnel barriers is challenging due to their strong dependence on the exact growth and lithography conditions, a challenge that for single-junction transmon devices has recently been addressed with individual laser-assisted annealing [20].

In this work, we introduce a wide range of singleJosephson-junction rf-SQUID qubits that rely on a geometric shunt inductor realized from a highly miniaturized approximately $50-\mu \mathrm{m}$-diameter planar aluminum coil suspended on a 220 -nm-thick silicon membrane - the device layer of a commercial silicon on insulator wafer. This new circuit element, introduced in Ref. [13], has low loss, behaves linearly, is easy to design and reproduce, and can be described as a lumped $L C$ circuit up to the frequency region of its first self-resonance. For a circular design with many turns $n \gg 1$ and a large filling factor, the capacitive energy $E_{C}=e^{2} /(2 C)$ is simply given by the outer radius of the coil and the inductive energy $E_{L}=\left(\Phi_{0} /(2 \pi)\right)^{2} / L$, where $\Phi_{0}$ is the magnetic flux quantum, by how many turns are fitted into that given radius. In contrast to previous implementations, such a geometric inductor is a simple two-terminal device that hosts a single uninterrupted superconducting wave function with a fixed and geometrically defined inductance and distributed capacitance, offering a high degree of control and design flexibility. Qubits made from such linear inductors are expected to be less sensitive to quasiparticles compared with those composed of disordered superconductors [21-23] owing to their small kinetic contribution $(<10 \%$ [13]) and because the lower disorder results in a shorter quasiparticle lifetime [14,24].

We show how the same physical circuit shown in Figs. 1(a) and 1(b) gives rise to different qubit types and qubit properties. Of the eight qubits studied, some reside in the classical flux qubit limit [29], characterized by a strong localization of phase with zero-point phase fluctuations of only $\varphi_{\mathrm{zpf}}=\left(2 E_{C} / E_{L}\right)^{1 / 4}=0.56$, while others reach the quasicharge regime [11] characterized by a strongly reduced flux sensitivity and $\varphi_{\mathrm{zpf}}=2.4$. This complements parallel work to delocalize the circuit groundstate phase by means of Cooper-pair cotunneling [30,31] and represents an important step toward realizing passively protected circuits [32-36] such as the $0-\pi[37,38]$ or the $\cos (2 \varphi)$ [39-41]. The qubit states of such devices would be spanned by two degenerate ground states protected by circuit symmetries and thus would rely on a very precise control of the qubit energies - properties that are ensured by top-down lithographically defined geometry in the present work.

\section{MODEL AND CLASSIFICATION}

This section highlights the variety of qubits that can be achieved with this first generation of geometric rf-SQUID circuits and the different physics that can be observed. We present results from eight different qubits that all derive from the circuit shown in Fig. 1(a) and are described by the Hamiltonian

$$
\hat{H}_{q}=4 E_{C} \hat{n}^{2}+\frac{1}{2} E_{L}\left(\hat{\phi}-2 \pi \Phi_{\text {ext }} / \Phi_{0}\right)^{2}-E_{J} \cos (\hat{\phi}),
$$

where $\hat{n}$ and $\hat{\phi}$ are the charge and phase operators, $E_{J}=$ $I_{c} \Phi_{0} /(2 \pi)$ is the Josephson energy, $\Phi_{\text {ext }}$ is the external flux, and $I_{c}$ the critical current of the single small Josephson junction. The potential is given by the second and third terms in Eq. (1) and the kinetic energy is given by the first term. It is important to note that Eq. (1) is the exact Hamiltonian of a Josephson junction shunted by an $L C$ circuit that is a faithful representation of the coil up to and above the first few qubit transitions [13]. The breakdown of the single-mode lumped-element model is described in Appendix B.

What makes this circuit interesting is the fact that drastically different physics and qubit properties can be realized only by changing the relative magnitude of the three energies $\left(E_{C}, E_{L}\right.$, and $\left.E_{J}\right)$ in the Hamiltonian. Figure 1(c) presents a classification scheme that is valid around the flux frustration point $\Phi_{\text {ext }}=0.5 \Phi_{0}$, where qubits are often operated due to the first-order protection from flux noise and the suppression of quasiparticle dissipation [25]. The axes represent the energy scales of the two potential terms $\left(E_{L}\right.$ and $\left.E_{J}\right)$ normalized by the energy of the kinetic term $\left(E_{C}\right)$.

On the $x$ axis, we have $E_{L} / E_{C} \propto 1 / Z_{C}^{2}$, where $Z_{C}=$ $\sqrt{L / C}$ is the characteristic impedance of the qubit. This quantity affects the quadratic part of the Hamiltonian and determines its overall steepness (gradient). When it is small, the phase is more delocalized since the wave functions of the ground and excited states bleed into more wells corresponding to higher zero-point phase fluctuation values. As the qubit wave function spreads in phase, the qubit transitions become less sensitive to flux noise.

On the $y$ axis, we plot $E_{J} / E_{C}$, which is a measure of the relative depth of the sinusoidal wells. The interwell coupling is exponentially dependent on $E_{J} / E_{C}$. Such coupling corresponds to the frequency of the qubit at the half-flux 
(a)

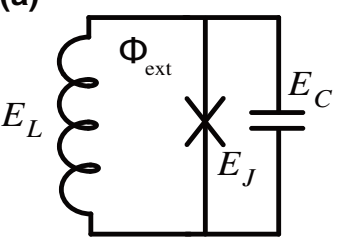

(b)

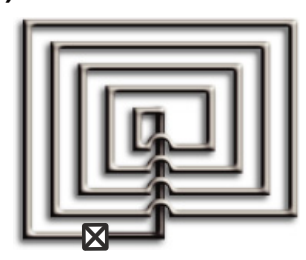

(c)

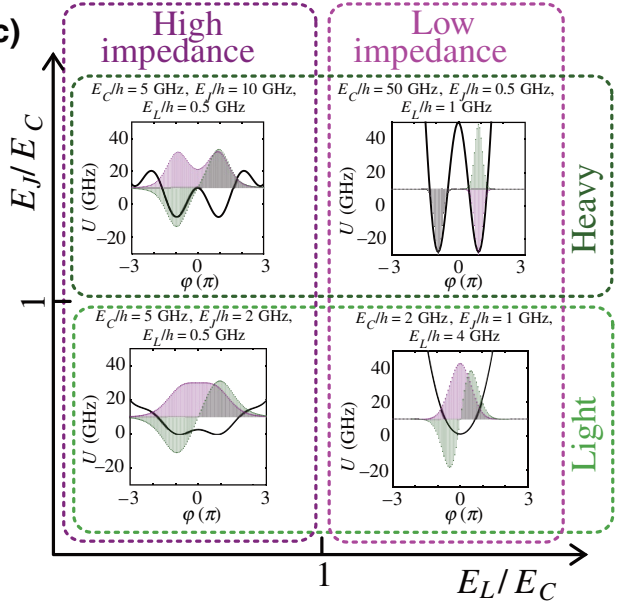

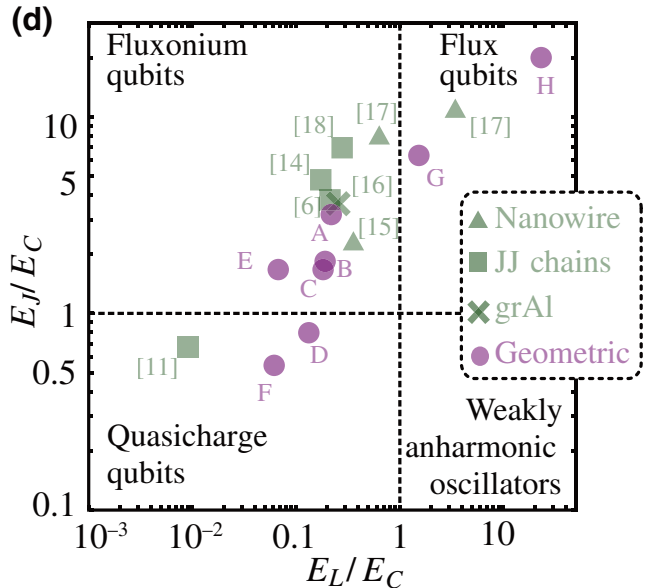

FIG. 1. The rf-SQUID qubit classification. (a) A rf-SQUID circuit with inductive energy $E_{L}$, Josephson energy $E_{J}$, capacitive energy $E_{C}$, and external flux $\Phi_{\text {ext }}$. (b) A sketch of a geometric rf-SQUID qubit - an exact implementation of the circuit in (a). (c) The classification scheme according to the ratios between the relevant energy scales at $\Phi_{\text {ext }}=0.5 \Phi_{0}$. Each quadrant shows the qubit potential $U$ as a function of the phase $\varphi$ superimposed with the ground-state (purple) and excited-state (green) wave functions (scaled for visibility). Different qubit limits are determined by whether the qubit characteristic impedance $Z_{C} \propto \sqrt{E_{C} / E_{L}}$ is large or small compared with the resistance quantum $R_{Q} \approx 6.45 \mathrm{k} \Omega$ and whether the qubit is heavy ( $E_{J} / E_{C}>1$, dominated by capacitance) or light $\left(E_{J} / E_{C}<1\right.$, dominated by tunneling). (d) The geometric rf-SQUID qubits presented in this paper (purple) alongside other qubits taken from the literature $[6,11,14,25-28]$ (green) placed within the classification presented in (c). The four classes, identified by their limit cases, are the fluxonium qubit, the flux qubit, the quasicharge qubit, and the weakly anharmonic oscillator limit. The extracted energies and design parameters for the geometric qubits are reported in Table I.

quantum, which can be made to dip down to $14 \mathrm{MHz}$ [28], allowing for large $T_{1}$ according to Fermi's golden rule for capacitive loss [42]. High- $E_{J} / E_{C}$ qubits are conventionally called "heavy" due to their low kinetic energy.

From these distinctions, four groups of qubits emerge that we classify according to their limit cases, which may be modeled with different Hamiltonians and as indicated in Fig. 1(d):

(1) The weakly anharmonic limit. These light and lowimpedance circuits display a potential very similar to that of an harmonic oscillator but with very faint deformations due to the Josephson term. This results in a qubit state that resembles very closely that of an $L C$ oscillator with a slight flux dispersion and a relatively small anharmonicity compared with its siblings. This limit might be interesting for a variety of applications including parametric amplification and for nonlinear-oscillator-based qubit implementations [43] but is not further studied in this work.

(2) The flux qubit limit. Here, qubits have a strongly localized wave function due to $E_{J}$ and $E_{L}$ being higher than $E_{C}$. When $E_{L}$ is large (close to $E_{J}$ ), the system is similar to the flux qubits made out of three

TABLE I. The qubit circuit parameters. The data include geometric properties such as the design type [two-dimensional (2D) or three-dimensional (3D)], the number of inductor turns $n$, the coil wire pitch $p$ and outer diameter $d_{\text {out }}$, and the total resonator line width $\kappa_{\text {tot }} / 2 \pi$, as well as fitted qubit energies $E_{L}, E_{C}$, and $E_{J}$ extracted from the data shown in Fig. 5 in Appendix A, the calculated zero-point fluctuations of the phase $\varphi_{\text {zpf }}$, the fitted coupling constants multiplied by the calculated matrix elements between states 0 and 1 at half flux, the measured values of $T_{1}$ also at half flux, and the fitted flux-noise amplitudes $A_{\phi}$.

\begin{tabular}{|c|c|c|c|c|c|c|c|c|c|c|c|c|c|}
\hline Qubit & Design & $n$ & $\begin{array}{c}p \\
(\mu \mathrm{m})\end{array}$ & $\begin{array}{c}d_{\text {out }} \\
(\mu \mathrm{m})\end{array}$ & $\begin{array}{c}\kappa_{\mathrm{tot}} /(2 \pi) \\
(\mathrm{MHz})\end{array}$ & $\begin{array}{c}E_{L} / h \\
(\mathrm{GHz}) \\
\end{array}$ & $\begin{array}{c}E_{C} / h \\
(\mathrm{GHz})\end{array}$ & $\begin{array}{c}E_{J} / h \\
(\mathrm{GHz}) \\
\end{array}$ & $\begin{array}{c}\varphi_{\mathrm{zpf}} \\
(\mathrm{rad})\end{array}$ & $\begin{array}{c}g_{C} \hat{n} /(2 \pi) \\
(\mathrm{MHz})\end{array}$ & $\begin{array}{c}g_{L} \hat{\phi} /(2 \pi) \\
(\mathrm{MHz})\end{array}$ & $\begin{array}{c}T_{1} \\
(\mu \mathrm{s}) \\
\end{array}$ & $\begin{array}{c}A_{\phi} \\
\left(\mu \Phi_{0}\right)\end{array}$ \\
\hline $\mathbf{A}$ & $2 \mathrm{D}$ & 74 & 0.4 & 66 & 1.7 & 0.618 & 2.75 & 8.55 & 1.73 & 15 & 0.1 & 1.5 & \\
\hline B & $2 \mathrm{D}$ & 74 & 0.4 & 66 & 0.63 & 0.620 & 3.15 & 5.92 & 1.78 & 63 & 140 & 2.38 & 317 \\
\hline $\mathrm{C}$ & $2 \mathrm{D}$ & 74 & 0.4 & 66 & 0.74 & 0.619 & 3.25 & 5.41 & 1.80 & 69 & 100 & 3.29 & 338 \\
\hline D & $2 \mathrm{D}$ & 74 & 0.4 & 66 & 0.62 & 0.620 & 3.83 & 3.05 & 1.88 & 41 & 210 & 1.81 & 787 \\
\hline $\mathbf{E}$ & $2 \mathrm{D}$ & 125 & 0.3 & 81 & 0.82 & 0.205 & 2.97 & 4.89 & 2.32 & 6 & 2 & 9.62 & 673 \\
\hline $\mathbf{F}$ & $2 \mathrm{D}$ & 125 & 0.3 & 81 & 0.95 & 0.215 & 3.40 & 1.99 & 2.42 & 90 & 7 & 2.25 & 646 \\
\hline $\mathbf{G}$ & $3 \mathrm{D}$ & 70 & 0.3 & 52 & 1.1 & 0.78 & 0.50 & 3.15 & 1.06 & 17 & 0 & $\ldots$ & $\ldots$ \\
\hline H & $3 \mathrm{D}$ & 25 & 0.25 & 17 & 0.95 & 10.70 & 0.54 & 9.00 & 0.56 & 98 & 0 & $\ldots$ & $\ldots$ \\
\hline
\end{tabular}


junctions in terms of spectrum and properties [29]. In the case of $E_{L}$ close to $E_{C}$, the physics is that of heavy fluxonium qubits, where the high value of $E_{J} / E_{C}$ reduces the charge-matrix element, making state transfer challenging and typically requiring the involvement of higher-order transitions [44] or nonadiabatic protocols [28] to prepare the first excited state at this flux value.

(3) The fluxonium qubit limit. Qubits in this limit have a high impedance and low interwell tunneling. These have been the first qubits to show phase delocalization by combining an rf SQUID with a superinductor [6]. It has been shown that in the limit of $E_{L} / E_{C} \ll 1$ and $E_{J} / E_{C} \gtrsim 2$, the lower energy levels and wave functions of this system are well described by a phase-slip box Hamiltonian $[45,46]$. The phase slip is the dual element of the Josephson junction and acts as a nonlinear capacitor [47]. In the limit of high $E_{J} / E_{C}$, however, the approximation breaks down, as many more levels come into play [45].

(4) The quasicharge qubit limit. These qubits are similar to the fluxonium qubit but due to low $E_{L}$ and low $E_{J}$ with respect to $E_{C}$, the wave function is able to spread further. In the case of very low $E_{L} / E_{C}$ [11], the spread of the wave function extends significantly beyond the two lowest potential wells, making the qubit more insensitive to flux noise than its siblings. However, due to low $E_{J} / E_{C}$, this qubit has relatively large matrix elements and hence the state is not well protected from relaxation.

In the following, we experimentally explore a large range spanning 2-3 orders of magnitude in both $E_{L} / E_{C}$ and $E_{J} / E_{C}$. Figure $1(\mathrm{~d})$ shows the parameters of qubits $\mathrm{A}-\mathrm{H}$ reported in this work alongside representative rf-SQUID qubits based on kinetic inductance.

\section{QUBIT DESIGNS}

Scanning electron micrographs (SEM) of a flux-type qubit $(\mathrm{G})$ and a quasicharge qubit $(\mathrm{F})$ are shown in Figs. 2(a) and 2(b). The flux qubit is placed in a threedimensional (3D) cavity, to which it couples via the large antenna that can be seen in the top inset. Such a coupling benefits from low dielectric loss and Purcell protection [25] but it adds considerable capacitance, making it incompatible with very light fluxonium devices. On the other hand, most devices in this study couple to a compact on-chip resonator and look like qubit F, shown in Fig. 2(b). In this case, both the qubit inductance (green) and the resonator (purple) are made out of geometric superinductors, which results in an extremely compact footprint of about $60 \mu \mathrm{m} \times 120 \mu \mathrm{m}$ for a full-circuit QED system.

The position of the two coils with respect to the coplanar waveguide coupler wire determines the external coupling
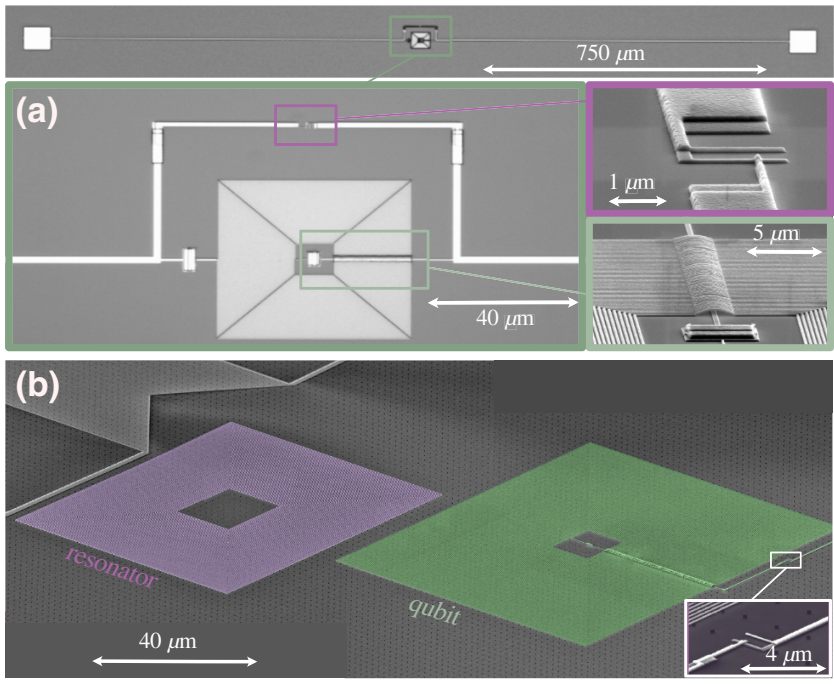

FIG. 2. Qubit designs and fabrication. (a) A SEM image of flux-type qubit $\mathrm{G}$ coupled to a three-dimensional (3D) cavity via the coupling antenna (top), which is fabricated from aluminum on high-resistivity silicon. The insets depict the planar coil inductor (green frame), with air bridges (bright green frame) and the Josephson junction (purple frame). (b) A false-color SEM image of quasicharge qubit $\mathrm{F}$ (green) with a single Josephson junction (inset) coupled to a resonator (purple), which is coupled to a shorted coplanar waveguide (top left). This device is fabricated from aluminum on a HF-vapor-etched silicon-on-insulator (SOI) wafer.

of the coils. The qubit coil is positioned symmetrically with respect to the coupler to minimize loss through this channel. The exact position is optimized in order to minimize the waveguide coupling. This still allows us to address the qubit without an additional drive line and without measurable heating of the dilution refrigerator at a repetition rate of $100 \mathrm{kHz}$. Qubit operations such as a $\pi$ rotation are performed with pulse lengths down to $20 \mathrm{~ns}$ and were limited by instrument rise times. The resonator coil, on the other hand, is asymmetrically positioned in order to obtain a $Q_{e} \approx 2 \times 10^{4}$ to facilitate the readout. Coupling between the qubit and the resonator is obtained via the mutual inductance as well as the cross-capacitance. For this work, the qubits are placed in close proximity to the resonator (2-5 $\mu \mathrm{m}$ distance) with the magnetic qubit-resonator coupling reaching as high as $210 \mathrm{MHz}$ for qubit D.

The qubit inductances are calculated analytically using Ref. [48] with a small kinetic inductance contribution [13] to be $280 \mathrm{nH}$ for qubits $\mathrm{A}-\mathrm{D}, 718 \mathrm{nH}$ for qubits E-F, 200 $\mathrm{nH}$ for qubit $\mathrm{G}$, and $29 \mathrm{nH}$ for qubit $\mathrm{H}$. The fitted values are found to be $264 \pm 0.4 \mathrm{nH}, 793 \pm 5 \mathrm{nH}, 209 \mathrm{nH}$, and $15 \mathrm{nH}$, respectively. Qubits that have the same coil geometry (qubits $\mathrm{A}, \mathrm{B}, \mathrm{C}$, and $\mathrm{D}$ as well as qubits $\mathrm{E}$ and $\mathrm{F}$ ) have a standard deviation of the inductance of only $0.2 \%$ and $0.7 \%$. This includes chip-to-chip variation, as qubits 
$\mathrm{A}$ and $\mathrm{D}$ are on a different chip to qubits $\mathrm{B}$ and $\mathrm{C}$, while $\mathrm{E}$ and $\mathrm{F}$ are fabricated months apart. This is at least an order of magnitude more accurate than the typical Josephson energy variation of a simple single-junction device without laser annealing and is compatible with the reproducibility requirements for symmetry protected or larger-scale devices. Even though Josephson-junction chains experience a reduction of the on-chip uncertainty by a factor of $\sqrt{N}$, where $N$ is the number of junctions, they typically display a higher chip-to-chip variation. For $E_{C}$, a simple formula is not available for square coils. Finiteelement simulations predict a capacitance of $2.77 \mathrm{fF}$ for the coils in qubits A-D and $3.21 \mathrm{fF}$ for the coils in qubits E-F. The fitted qubit capacitances, which include the junction and coupling capacitances, are found to be $5.9 \pm 1 \mathrm{fF}$ and $6.1 \pm 0.6$ for the two designs, respectively. Here, the capacitance variations are mainly due to Josephson-junction size and coupling design differences.

\section{QUBIT SPECTRA}

So far, the rf-SQUID qubits have been coupled to a resonator either capacitively or inductively. The type of coupling is often trivially given by the qubit geometry; for example, a shared inductance will give an inductive coupling while a large antenna placed in a 3D cavity will produce a capacitive coupling. While the flux qubits (qubits $\mathrm{G}$ and $\mathrm{H}$ ) are capacitively coupled to a $3 \mathrm{D}$ cavity via an antenna, in the case of the other qubits explored in this paper, neither coupling type can be completely excluded. We model this situation by adding both interaction terms in the full-system Hamiltonian

$$
\hat{H}=\hat{H}_{q}+\hbar \omega_{r} \hat{a}^{\dagger} \hat{a}-i \hbar g_{C} \hat{n}\left(\hat{a}-\hat{a}^{\dagger}\right)-\hbar g_{L} \hat{\phi}\left(\hat{a}+\hat{a}^{\dagger}\right),
$$

where $\omega_{r}$ is the resonator angular frequency and $\hat{a}\left(\hat{a}^{\dagger}\right)$ is the annihilation (creation) operator. The third and fourth terms are the capacitive and inductive coupling, where $g_{C}$ $\left(g_{L}\right)$ is the capacitive (inductive) coupling constant.

The fitting of fluxonium spectra and their coupling constants can be a challenge due to the multitude of parameters and their nontrivial effect on the energy levels. In an effort to make parameter fitting computationally tractable, the energy levels of the full Hamiltonian are computed using the in-place eigenvalue solvers for Hermitian matrices available in the JULIA standard library. These solvers are highly performant, yielding eigenvalues for a $100 \times$ 100 Hermitian matrix (five photon states and 20 plasmon states) in less than $2 \mathrm{~ms}$ on a typical laptop (Intel Core i7-6700HQ CPU @ 2.60 GHz × 8). The eigenvalue solver is then run repeatedly by a gradient-free local optimization routine [49] until it converges on a set of parameters that best matches the data. Since the optimization routine is local, whether a trajectory converges on the correct solution depends on the choice of initial parameters. The most efficient strategy is to first optimize the qubit parameters $\left(E_{L}, E_{C}\right.$, and $\left.E_{J}\right)$ to Eq. (1) and then add the coupling constants $\left(g_{L}\right.$ and $\left.g_{C}\right)$ and fit to the full model while fixing values for qubit parameters. The code implementing the fitting of rf-SQUID spectra and coupling constants has been made available [50].

Figures 3(a)-3(c) show spectra from three different qubits that reside in three of the categories presented in Fig. 1(c). The external flux is provided by a centimeter-sized bias coil attached to the outer side of the copper box containing the sample. Tuning of one flux quantum is obtained by sweeping the current by $1 \mu \mathrm{A}$ on average. This results in an approximate external magnetic field of $2 \times 10^{-3} \mathrm{G}$, in agreement with the coupling being proportional to the outer area of the qubit loop. Above each qubit spectrum, the resonator dispersion versus the flux is shown. These are reflective measurements taken with a vector network analyzer, while the spectroscopy data are obtained with two-tone spectroscopy. The resonators of qubits $\mathrm{G}$ and $\mathrm{F}$ have a relatively low variation in frequency due to a low coupling constant in the case of $\mathrm{G}$ and due to small flux dispersion for qubit $\mathrm{F}$. On the other hand qubits A, B, C, and $\mathrm{D}$ have high coupling constants and large flux dispersions $(4-6 \mathrm{GHz})$ and therefore all display anticrossings and large frequency variations in the dispersive shift. The quasicharge qubit is the one with the smallest flux dispersion, this being due to the wave function residing in multiple wells at once. This qubit is comprised of one of the largest coils with the largest coil capacitance and inductance, lowering the frequency of the coil parasitic mode down into the accessible measurement range. Quantitative modeling of the parasitic mode coupling is presented in Appendix B.

A challenge arises in finding the correct coupling constants when trying to fit the flux-dependent resonance shift, shown in the top panels of Fig. 3. Out of the three predominant quantities (namely $g_{L}, g_{C}$, and the resonator bare frequency $\omega_{r}$ ), only two are independent. This implies that there are a multitude of potential solutions. We narrow this down to one solution by fixing the bare resonator frequency, which is measured separately at high probe power. The plots show excellent agreement with the diagonalized Hamiltonian. In addition to the bare qubit transitions, many additional lines appear in the spectroscopy: these lines can easily be traced to being qubit transitions connected to a higher photon number. One of these transitions can be seen in Fig. 3(b). All fitted qubit and coupling parameters are reported in Table I, and all measured and fitted spectra are presented in Appendix A.

For qubits $\mathrm{B}, \mathrm{C}$, and $\mathrm{D}$ - due to a relatively high transition frequency at the flux sweet spot and large qubitresonator coupling - the dispersive shift is larger than the qubit line width, which leads to photon-number splitting of the qubit spectroscopy measurement similar to that observed for transmon qubits [51]. Measurements 
(a)
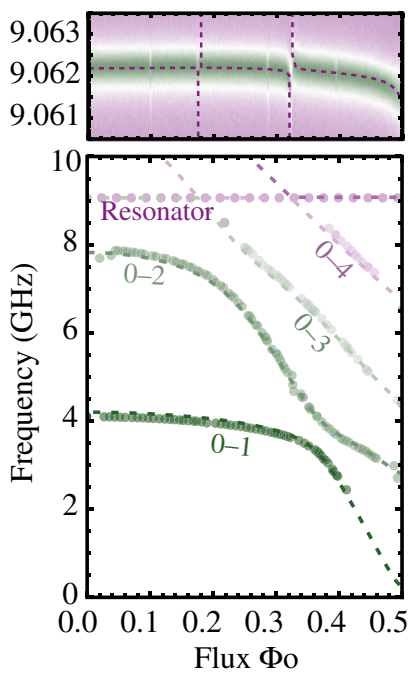

(b)
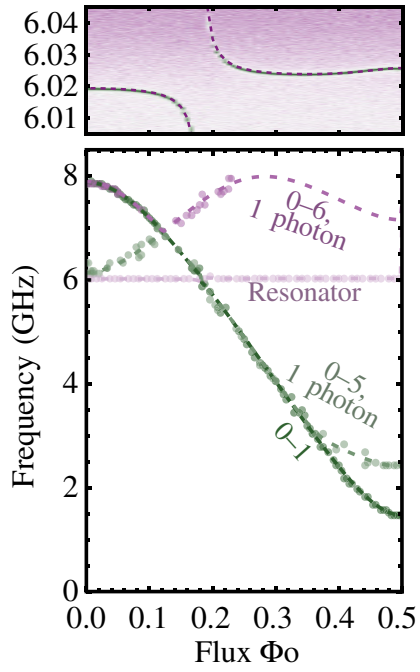

(c)
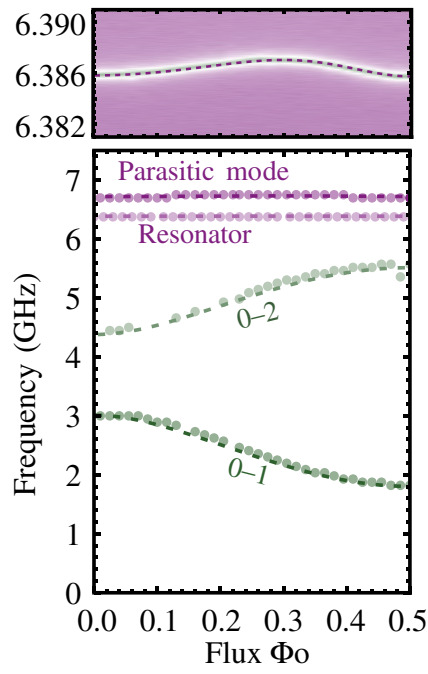

FIG. 3. Flux-dependent qubit and resonator spectra. The measured resonator reflection amplitude (top, color scale) and two-tone spectroscopy (bottom, dots) of three representative qubits as a function of the flux in (a) a flux qubit (G), (b) a fluxonium qubit (C), and (c) a quasicharge qubit (F). The dotted lines are fits to the diagonalized spectrum of the full-system Hamiltonian given by Eq. (2). Only in the case of the quasicharge qubit in (c) is the qubit coil parasitic mode lowered significantly, due to a very large coil inductance and capacitance; this becomes visible in the spectroscopy measurement at $6.7 \mathrm{GHz}$. A quantitative model of the parasitic mode is presented in Appendix B.

of photon-number-resolved fluxonium spectroscopy are shown in Appendix C.

\section{TIME-DOMAIN ANALYSIS}

In this section, we focus on the time-domain results of a quasicharge qubit (qubit F) and a fluxonium qubit (qubit E). Measured time-domain data from other qubits are summarized in Table I. These two qubits are chosen as they have the same design, their only difference being the value of $E_{J}$. This difference, however, has strong implications for the qubit dynamics. Qubit $\mathrm{E}$ has a larger flux dispersion in the $0-1$ qubit transition and hence is more susceptible to flux noise. However, due to the higher $E_{J}$, the qubit state is better protected from radiative decay due to a higher tunneling barrier.

The effects of these characteristics are visible in Fig. 4(a), which shows the measured $T_{1}$ values multiplied by the absolute square of the phase matrix element as a function of the qubit transition frequency. Multiplying by the matrix element allows us to compare the relaxation of qubits with different energies. The $T_{1}$ data are extracted by placing the qubit in a mixed state via a saturation pulse and then measuring its decay with a dispersive readout. The extracted values are consistent with measurements done with a short (25-30-ns) $\pi$-pulse excitation at the flux sweet spot. The matrix elements are calculated numerically using the SCQUBITS PYTHON library [52].

The behavior of the data agrees with a pure capacitive loss model, indicating that other loss mechanisms such as inductive loss or the Purcell effect are not limiting. Even with the high coupling, the Purcell limit to $T_{1}$ for these qubits is in the hundreds of microsecond range due to the
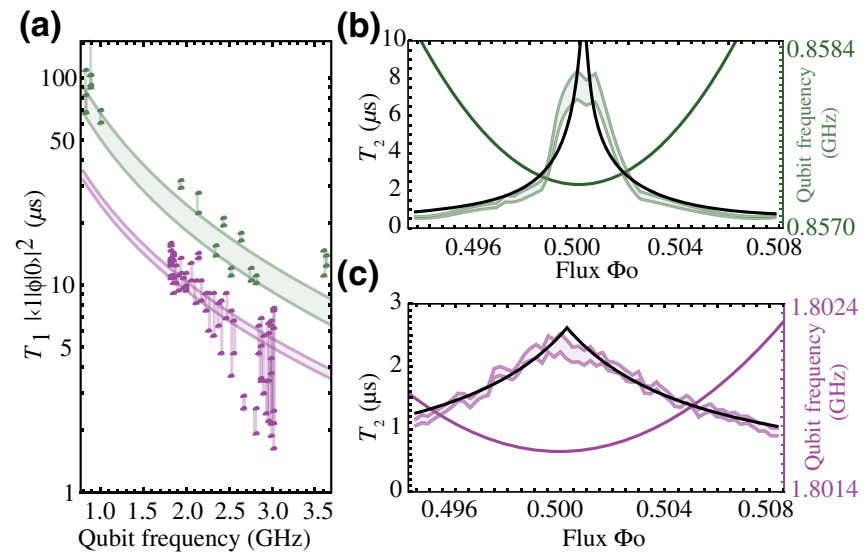

FIG. 4. The time-domain loss analysis. (a) The measured energy-relaxation time multiplied by the flux matrix element squared $T_{1}|\langle 0|\hat{\phi}| 1\rangle|^{2}$ of the fluxonium qubit $\mathrm{E}$ (green) and the quasicharge qubit $\mathrm{F}$ (purple) as a function of the external-fluxcontrolled qubit frequency. The bands are fits to the dielectric loss model given in Eq. (3), with the fit parameter $Q_{\text {diel }}$ for qubits $\mathrm{E}$ and F. (b),(c) The measured coherence time $T_{2}^{\text {echo }}$ as a function of the external flux around half flux for qubits $\mathrm{E}$ and $\mathrm{F}$. The bands represent the $90 \%$ confidence interval of the spin-echo sequence fit: the black line is a fit to the flux-noise decoherence model given in Eq. (4) and the purple (green) solid lines show the calculated qubit transition frequency (right axis). 
large detuning. We plot the data alongside a temperaturedependent capacitive loss model derived from Fermi's golden rule [42]:

$$
\Gamma_{1}=1 / T_{1}=\frac{1}{(2 e)^{2}}|\langle 0|\hat{\phi}| 1\rangle|^{2} \hbar \omega_{q}^{2} \frac{C}{Q_{\text {diel }}} \operatorname{coth}\left(\frac{\hbar \omega}{2 k_{B} T}\right),
$$

where $\Gamma_{1}$ is the relaxation rate, $T_{1}$ is the relaxation time, $\langle 0|\hat{\phi}| 1\rangle$ is the phase matrix element, $\omega_{q}$ is the angular frequency of the qubit, $C$ and $Q_{\text {diel }}$ are the total capacitance of the qubit and its quality factor, and $T$ is the qubit temperature.

The bands shown in Fig. 4(a) are fits to Eq. (3), with a fitted $Q_{\text {diel }}$ of $(57 \pm 8) \times 10^{3}$ for the fluxonium qubit and $(25 \pm 1) \times 10^{3}$ for the quasicharge qubit. These values are similar to quality factors found for geometric superinductor resonators at single-photon power on the same substrate (i.e., without handle-wafer removal) [13] and on par with other fluxonium implementations [27] but 10-100 times lower than state-of-the-art implementations $[7,25]$ and similar coils with the handle wafer removed [13]. This is likely due to the high surface participation ratio or the low resistivity of the commercial silicon-oninsulator (SOI) wafer. The temperature is taken as $60 \mathrm{mK}$ and $80 \mathrm{mK}$, respectively, these numbers derive from the fit to the coherence data explained below. The values of $T_{1}$ of the fluxonium qubit are found to be consistently higher than the values for the quasicharge qubit. This is in part due to a lower matrix element, which stems from the larger tunneling barrier and a higher quality factor due to differences in fabrication.

Compared to state-of-the-art coherence-optimized superconducting qubit devices, the observed values of $T_{1}$ are moderate in this first generation of geometric rf-SQUID qubits. However, these are obtained on an extremely small footprint with gap sizes as small as $150 \mathrm{~nm}$, which naturally leads to a higher sensitivity to two-level fluctuatorinduced loss. Better materials such as NbTiN or Ta and/or suitable surface treatments together with design optimizations that also include removal of the handle wafer, as in Ref. [13] should lead to a significantly longer $T_{1}$ in the near future.

Figures 4(b) and 4(c) show $T_{2}^{\text {echo }}$ as a function of the flux in the vicinity of the sweet spot for the same two qubits. The purple and green bands represent the coherence times and errors extracted from separate measurements. The black solid line represents the fit to a flux-noise-induced decoherence model with an upper bound of $2 T_{1}$ :

$$
\Gamma_{2}^{\text {echo }}=1 / T_{2}^{\text {echo }}=\frac{\partial \omega_{q}}{\partial \Phi} \sqrt{A_{\Phi}} \gamma+\frac{1}{2 T_{1}}+\frac{1}{T_{\phi}},
$$

where $\Gamma_{2}^{\text {echo }}$ is the decoherence rate, $T_{2}^{\text {echo }}$ is the coherence time, $\Phi$ is the flux, $A_{\Phi}$ is the noise amplitude of the flux-noise spectral density $S_{\Phi}(\omega)=A_{\Phi} / \omega, \gamma$ is a constant that depends on the specific filtering function given by the chosen spin-echo sequence, and $T_{\phi}$ is a phenomenological constant added to account for photon shot noise due to the strong coupling to the resonator. This latter effect is exacerbated when the dispersive cavity shift is larger than the cavity line width [53], which is the case for qubits A-F.

The difference between the two types of qubits can be seen in how quickly the values of $T_{2}^{\text {echo }}$ decay as the flux is tuned away from the sweet spot. The dependence on the derivative of the transition frequency $\partial \omega_{q} / \partial \Phi$ stresses the advantage of making a quasicharge qubit. For the two qubits presented in Figs. 4(b) and 4(c), we find $\sqrt{A_{\Phi}}$ to be $646 \mu \Phi_{0}$ and $673 \mu \Phi_{0}$, respectively. This is 2 orders of magnitude higher than what is measured in SQUIDs [54] and other fluxonium devices [7]. We attribute this to the long perimeter of the coil, since the flux-noise amplitude is likely due to magnetic spin defects at the surface and is therefore perimeter dependent [54]. The shot-noise contribution is fitted to be $30.0 \mu \mathrm{s}$ and $6.2 \mu \mathrm{s}$ for qubits $\mathrm{E}$ and $\mathrm{F}$, respectively. These losses correspond to 0.03 and 0.006 average thermal photons left over in the cavity, i.e., a thermal bath of $80 \mathrm{mK}$ and $60 \mathrm{mK}$ for the two devices characterized in separate measurement runs. These numbers are comparable with similar implementations [29] and could be further improved with better shielding.

\section{CONCLUSIONS AND OUTLOOK}

In this work, we update the classification of rf-SQUID qubits $[55,56]$ to include the several recent additions with a focus on the physics [11,36] rather than the specific physical implementation, which often defines a new qubit. This is guided by the possibility of fabricating superinductors that are free of disorder and islands and at the same time give access to a characteristic energy parameter range spanning 3 orders of magnitude, from a highly localized to a strongly delocalized qubit phase wave function.

The measured quasicharge qubit spectrum provides direct evidence that the deep-superinductor regime can be unlocked without significant kinetic inductance in practical devices. This refutes the broadly accepted hypothetical vacuum-impedance limitation for conventional inductors and establishes miniaturized planar coils as a highimpedance linear superconducting circuit element, with applications ranging from metrology, sensing, metamaterials, and hybrid devices to quantum information processing.

As a result of the geometric inductance approach, we observe simultaneous capacitive and inductive coupling between the qubit and the resonator and we provide a model and an efficient algorithm that can fit the coupled rf-SQUID-resonator spectrum. We find couplings of the order of tens to hundreds of megahertz achieved with a very small coupling capacitance of only $1-2 \mathrm{fF}$ due to the small size and high impedance of both the qubit and the 
resonator - a feature that enables large coupling in the light fluxonium and quasicharge regimes.

While the linearity of the inductor and its suspension on a thin silicon membrane are expected to lead to a significantly lower quasiparticle loss limit, the observed high flux-noise amplitude as a result of the long SQUID wire is a potential disadvantage. This highlights the need for low flux dispersion by design, as achieved in the case of the measured quasicharge qubit, where $T_{2}$ is limited by $T_{1}$ and the calculated shot noise. Other mitigation strategies include further miniaturization of the coil geometry in order to maximize the inductance per unit length as well as the use of materials with fewer magnetic-surfacedefect states. On the positive side, the different susceptibility of geometric superinductors to quasiparticles and flux noise with respect to their kinetic counterparts opens the door to studying the origin of these effects in more detail and developing better materials and fabrication processes, which will ultimately be beneficial to all superconducting qubit types. Furthermore, the increased flux sensitivity points to other potential applications, such as highprecision quantum sensing of elementary spin systems.

The coherence and relaxation time can be improved with more optimized design choices in future device generations - most importantly, by reducing the two level systems loss of the inductor; for example, by back-etching the handle wafer [13], which would incidentally also allow us to reach even lower values of $E_{L} / E_{C}$. The resulting further enhanced zero-point phase fluctuations are a prerequisite toward the realization of degenerate groundstate qubits, where the full protection requires Hamiltonian engineering with carefully maintained circuit symmetries, a characteristic of top-down fabricated circuit elements.

The high reproducibility of the inductive energy together with the extremely small circuit size of only $100 \times 150 \mu \mathrm{m}^{2}$ for qubit and resonator could also render the geometric qubit approach advantageous for scaling, given that the coherence can be improved sufficiently on such a small footprint. The high-power handling of geometric superinductors as well as the quadratic confinement provided by the inductive shunt (compared with the unconfined potential of the transmon [57]) should, furthermore, allow for higher-power readout and larger-amplitude driven-dissipative quantum error-correction protocols to be implemented.

In summary, we believe that the demonstrated design flexibility, the low chip-to-chip variance of the inductive energy of $<1 \%$ in different fabrication runs, the simplicity of predicting the physics of two-terminal devices, the ability to reach very high inductance values, and the capability of controllable magnetic coupling to resonators, waveguides, or other qubits make geometric superinductor qubits an interesting and complementary ingredient toward realizing hardware-protected qubits in new parameter regimes in the near future.
The data and code used to produce the figures in this manuscript will be made available at Zenodo [58]

\section{ACKNOWLEDGMENTS}

We thank W. Hughes for analytic and numerical modeling during the early stages of this work, J. Koch for discussions and support with the SCQUBITS package, R. Sett, P. Zielinski, and L. Drmic for software development, and G. Katsaros for equipment support, as well as the MIBA workshop and the Institute of Science and Technology Austria nanofabrication facility. We thank I. Pop, S. Deleglise, and E. Flurin for discussions. This work was supported by a NOMIS Foundation research grant, the Austrian Science Fund (FWF) through BeyondC (F7105), and IST Austria. M.P. is the recipient of a Pöttinger scholarship at IST Austria. E.R. is the recipient of a DOC fellowship of the Austrian Academy of Sciences at IST Austria.

\section{APPENDIX A: SPECTRA OF ALL QUBITS}

The spectroscopy data of all qubits can be found in Fig. 5. These show a clean spectrum, where most lines can be attributed to qubit or dressed states. The parameters corresponding to the fits are reported in Table I alongside geometric parameters of the samples and the time-domain measurement results that are conducted.

\section{APPENDIX B: COUPLING TO THE PARASITIC MODE}

The planar coil used as a superinductor is a distributedelement circuit. At low frequencies, it can be described by a simple lumped-element model, i.e., a parallel $L C$ circuit, where the fundamental mode forms the first transition frequency of the qubit. The second mode of the coil inductor acts as a resonator mode that couples strongly to this qubit mode. We use finite-element simulations of the planar-coil inductor used for qubits $\mathrm{E}$ and $\mathrm{F}$ to predict the frequency of this mode, as shown in Fig. 6(a). At a frequency of about $6.5 \mathrm{GHz}$, the admittance shows an additional pole compared to the simple $L C$ model. We model this by adding, in parallel to the original $L C$ circuit, an extra inductance and capacitance in series, as shown in Fig. 6(b), which agrees well with the simulated values. In the future, this mode could therefore potentially be used as a built-in readout resonator to further simplify the circuit design.

The Lagrangian for this circuit is

$$
\begin{aligned}
\mathcal{L}= & C_{q}\left(\dot{\phi}_{q}\right)^{2}+C_{p}\left(\dot{\phi}_{q}-\dot{\phi}_{p}\right)^{2}+\frac{1}{2} E_{L, q} \phi_{q}^{2} \\
& +\frac{1}{2} E_{L, p} \phi_{p}^{2}-E_{J} \cos \phi_{q},
\end{aligned}
$$

where $C_{q}\left(C_{p}\right)$ and $\phi_{q}\left(\phi_{p}\right)$ represent the capacitance and phase variable of the qubit (parasitic mode) and $E_{L, q}$ and 
(a)

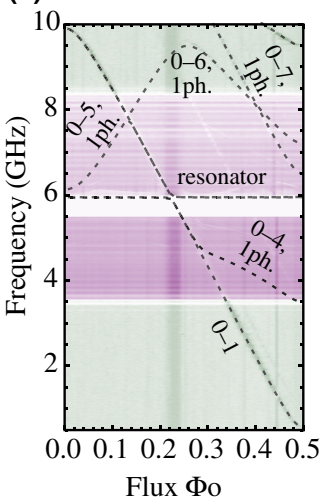

(e)

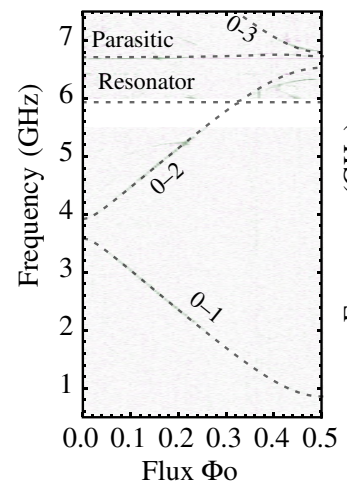

(b)

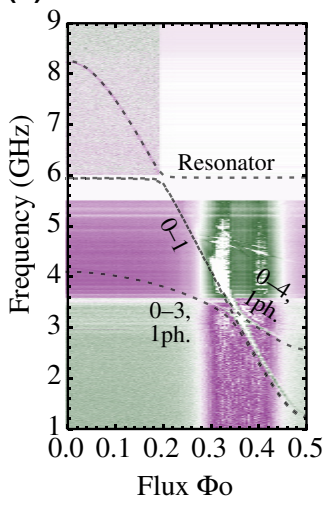

(f)

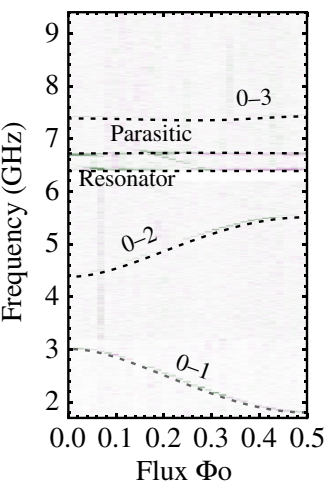

(c)

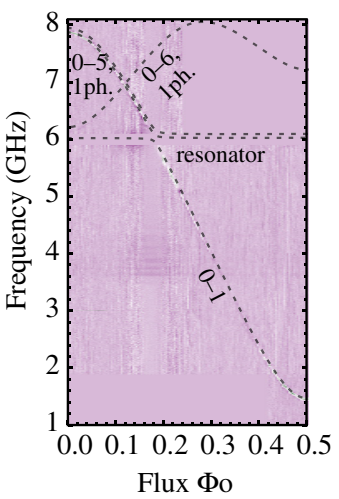

(g)

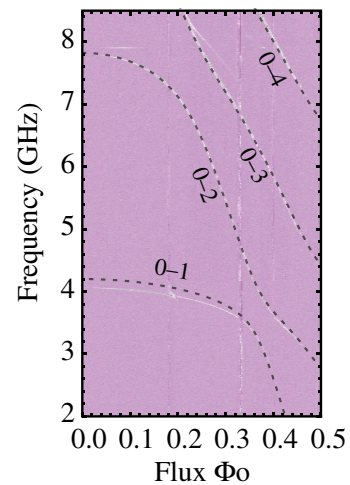

(d)

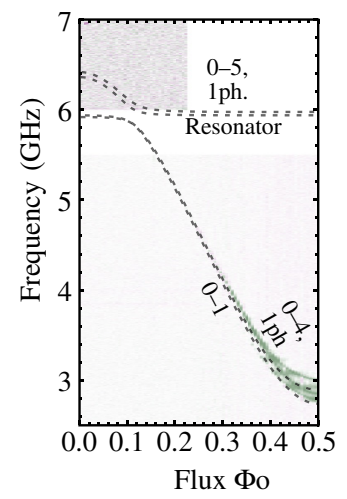

(h)

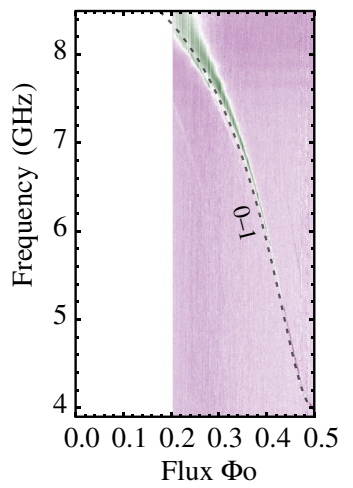

FIG. 5. The two-tone spectroscopy of all measured qubits. The labeled lines are obtained from a numerical optimization algorithm of the qubit and coupling parameters to maximize agreement with the full rf-SQUID and resonator model given in Eq. (2). In the case of qubits $\mathrm{E}$ and $\mathrm{F}$, the observed parasitic qubit mode is added as a second coupled-resonator mode to the Hamiltonian.

$E_{L, p}$ represent the inductive energies of the qubit and parasitic mode, corresponding to $L_{q}$ and $L_{p}$ as shown in Fig. 6(b). Next, the relation between the node voltages and currents of the circuit must be identified. This enables the replacement of the node voltages $\dot{\phi}_{q}$ and $\dot{\phi}_{p}$ with their respective canonical conjugates $Q_{q}=\partial I_{q} t$ and $Q_{p}=$ $\partial I_{p} t$. Using Kirchhoff's laws, one can write the following equations in the Fourier domain:

$$
\left\{\begin{array}{l}
V_{q}-V_{p}=\frac{I_{p}}{C_{p} S}, \\
V_{q}=\frac{I_{q}-I_{p}}{C_{p} S}
\end{array}\right.
$$

where $S$ is the complex frequency, which corresponds to a differentiation operator in the time domain $d / d t$, while $1 / S$ is an integration operator $\int d t$.

Using these relations and the fact that $V_{q}=\dot{\phi}_{q}$ and $V_{p}=$ $\dot{\phi}_{p}$, we can rewrite Eq. (B2) as

$$
\left\{\begin{array}{l}
\dot{\phi_{p}}=\frac{Q_{q}-Q_{p}}{C_{q}}-\frac{Q_{p}}{C_{p}} \\
\dot{\phi}_{q}=\frac{Q_{q}-Q_{p}}{C_{q}} .
\end{array}\right.
$$

Substituting Eq. (B3) into Eq. (B1) gives rise to the final form of the total Hamiltonian:

$$
\begin{aligned}
H= & H_{\text {parasitic }}+H_{\text {qubit }}+H_{\text {coupling }} \\
= & \frac{\hat{Q}_{p}^{2}}{\left(C_{p}^{-1}+C_{q}^{-1}\right)^{-1}}+\frac{1}{2} E_{L, p} \hat{\phi}_{p}^{2}+\frac{1}{2} E_{L, q} \hat{\phi}_{q}^{2}-E_{J} \cos \hat{\phi}_{q} \\
& +\frac{\hat{Q}_{q}^{2}}{C_{q}}+\frac{2 \hat{Q}_{q} \hat{Q}_{p}}{C_{q}}
\end{aligned}
$$

where we quantize $\hat{\phi}$ and $\hat{Q}$. From Eq. (B4), it is possible to identify the frequency of the parasitic mode as

$$
\omega_{p}=\sqrt{\frac{1}{L_{p}\left(C_{p}^{-1}+C_{q}^{-1}\right)^{-1}}} .
$$


(a)
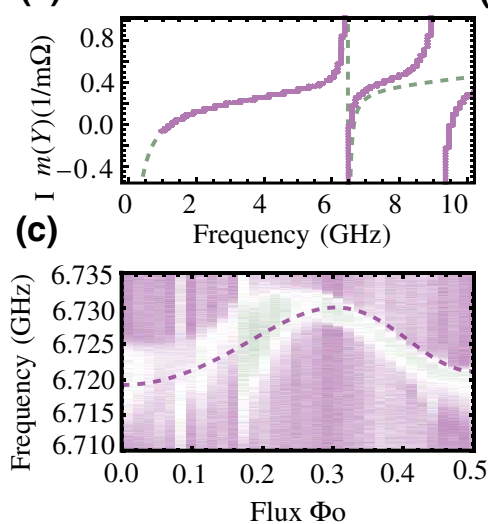

(b)

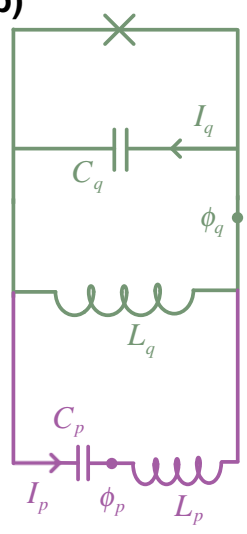

FIG. 6. The qubit parasitic mode. (a) The simulated admittance of the coil used in qubits $\mathrm{E}$ and $\mathrm{F}$. The purple dots represent the simulation, while the green dashed line is a fit to the circuit shown in (b). The fitted parameters are $C_{q}=4.8 \mathrm{fF}, L_{q}=530$ $\mathrm{nH}, C_{p}=0.47 \mathrm{fF}$, and $L_{p}=1.3 \mu \mathrm{H}$. The simulated data show an additional pole appearing at higher frequency that was not identified in the qubit spectrum. (b) A phenomenological circuit model for the high-frequency response of the coil inductor that shows very good agreement with the simulated admittance up around $8 \mathrm{GHz}$. The green part of the circuit represents the qubit, while the purple part models the parasitic mode. (c) The two-tone spectroscopy data of the parasitic mode of the qubit. The dashed line is obtained by solving the full Hamiltonian containing the qubit, the resonator mode, and the parasitic mode. Here, the coupling to the parasitic mode is taken to be $0.84 \mathrm{GHz}$, as correctly predicted by Eq. (B7).

The rewriting of the coupling term of Eq. (B4) in the second quantization formalism results in

$$
\begin{aligned}
H_{\text {coupling }} & =\frac{2 \hat{Q}_{q} \hat{Q}_{p}}{C_{q}}=\frac{2 \hat{Q}_{q}}{C_{q}}\left(\hat{a}+\hat{a}^{\dagger}\right) \sqrt{\frac{\hbar}{2 Z_{p}}} \\
& =\frac{2}{C_{q}} \sqrt{\frac{\hbar}{2 Z_{p}}}\left(\hat{a}+\hat{a}^{\dagger}\right) \hat{Q}_{q},
\end{aligned}
$$

which identifies the coupling strength as

$$
g_{p}=\frac{4 e}{C_{q}} \sqrt{\frac{\hbar \omega_{p}\left(C_{p}^{-1}+C_{q}^{-1}\right)^{-1}}{2}} .
$$

By fitting the coil admittance found in Fig. 6(a), we find the parasitic inductance to be $L_{p}=1.28 \mu \mathrm{H}$ and the parasitic capacitance to be $C_{p}=0.47 \mathrm{fF}$. By inserting these values into Eq. (B5) alongside the fitted qubit parameters, the frequency of the parasitic mode is expected to be at $6.74 \mathrm{GHz}$, very close to the measured value seen in Fig. $6(\mathrm{c})$, and the coupling is expected to be $g_{p}=0.84 \mathrm{GHz}$. The fit line in Fig. 6(c) is obtained by diagonalizing the Hamiltonian of the whole system consisting of the qubit,

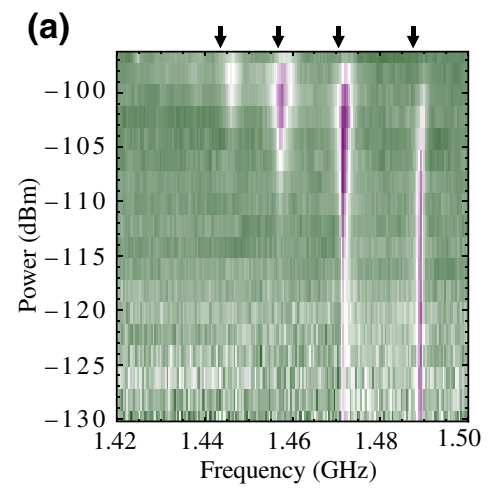

(b)

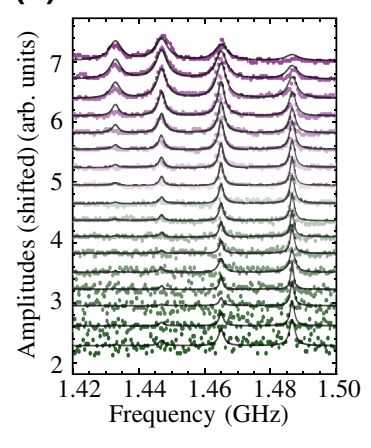

FIG. 7. The measured photon-number splitting. (a) The spectroscopy of qubit $\mathrm{C}$ as a function of the measurement power. The arrows indicate the solution of the full Hamiltonian. (b) Individual measurement traces from (a) plotted with an offset for visibility. The black lines correspond to fits to a Poissonian weighted sum of Lorentzians.

the resonator, and the parasitic mode, where the parasitic mode is added as an additional resonator coupled to the qubit. For the fit, the frequency of the parasitic mode is taken to be $6.73 \mathrm{GHz}$, while the coupling is taken from Eq. (B7), with values based on the simulated admittance.

\section{APPENDIX C: FLUXONIUM PHOTON-NUMBER SPLITTING}

Due to the large coupling strengths of qubits B, C, and $\mathrm{D}$, they all show some form of resolved photon-number splitting at both the sweet spots of the first qubit transition. Figure 7 shows the effect measured in qubit $\mathrm{C}$ as a function of the resonator measurement power. The resonators for these qubits are all found around $6 \mathrm{GHz}$ - specifically, $6.03 \mathrm{GHz}$ in the case of qubit C. Figure 7(a) shows a 2D plot of the measurement. We observe that the separation between the photon-number-resolved spectroscopy peaks is in agreement with the full Hamiltonian diagonalization. Figure 7(b) shows the individual traces from Fig. 7(a) offset for better visibility and fitted to a series of Lorentzians, the amplitudes of which are fixed by a Poisson distribution. In this measurement, we observe a sizable single-photon excitation probability of the resonator, indicating that an improved shielding is necessary to avoid excess qubit dephasing. Such improvements are implemented for later measurements of qubits $\mathrm{E}$ and $\mathrm{F}$.

[1] J. Clarke and F. K. Wilhelm, Superconducting quantum bits, Nature 453, 1031 (2008).

[2] U. Vool and M. Devoret, Introduction to quantum electromagnetic circuits, Int. J. Circ. Theory Appl. 45, 897 (2017). 
[3] P. Krantz, M. Kjaergaard, F. Yan, T. P. Orlando, S. Gustavsson, and W. D. Oliver, A quantum engineer's guide to superconducting qubits, Appl. Phys. Rev. 6, 021318 (2019).

[4] A. Blais, A. L. Grimsmo, S. Girvin, and A. Wallraff, Circuit quantum electrodynamics, Rev. Mod. Phys. 93, 025005 (2021).

[5] A. Gyenis, A. Di Paolo, J. Koch, A. Blais, A. A. Houck, and D. I. Schuster, Moving beyond the transmon: Noiseprotected superconducting quantum circuits, PRX Quantum 2, 030101 (2021).

[6] V. E. Manucharyan, J. Koch, L. I. Glazman, and M. H. Devoret, Fluxonium: Single Cooper-pair circuit free of charge offsets, Science 326, 113 (2009).

[7] L. B. Nguyen, Y.-H. Lin, A. Somoroff, R. Mencia, N. Grabon, and V. E. Manucharyan, High-Coherence Fluxonium Qubit, Phys. Rev. X 9, 041041 (2019).

[8] N. Earnest, S. Chakram, Y. Lu, N. Irons, R. K. Naik, N. Leung, L. Ocola, D. A. Czaplewski, B. Baker, J. Lawrence, J. Koch, and D. I. Schuster, Realization of a $\Lambda$ System with Metastable States of a Capacitively Shunted Fluxonium, Phys. Rev. Lett. 120, 150504 (2018).

[9] A. Somoroff, Q. Ficheux, R. A. Mencia, H. Xiong, R. V. Kuzmin, and V. E. Manucharyan, Millisecond coherence in a superconducting qubit, ArXiv:2103.08578 (2021).

[10] J. Koch, T. M. Yu, J. Gambetta, A. A. Houck, D. I. Schuster, J. Majer, A. Blais, M. H. Devoret, S. M. Girvin, and R. J. Schoelkopf, Charge-insensitive qubit design derived from the Cooper pair box, Phys. Rev. A 76, 042319 (2007).

[11] I. V. Pechenezhskiy, R. A. Mencia, L. B. Nguyen, Y.-H. Lin, and V. E. Manucharyan, The superconducting quasicharge qubit, Nature 585, 368 (2020).

[12] N. A. Masluk, I. M. Pop, A. Kamal, Z. K. Minev, and M. H. Devoret, Microwave Characterization of Josephson Junction Arrays: Implementing a Low Loss Superinductance, Phys. Rev. Lett. 109, 137002 (2012).

[13] M. Peruzzo, A. Trioni, F. Hassani, M. Zemlicka, and J. M. Fink, Surpassing the Resistance Quantum with a Geometric Superinductor, Phys. Rev. Appl. 14, 044055 (2020).

[14] L. Grünhaupt, M. Spiecker, D. Gusenkova, N. Maleeva, S. T. Skacel, I. Takmakov, F. Valenti, P. Winkel, H. Rotzinger, W. Wernsdorfer, A. V. Ustinov, and I. M. Pop, Granular aluminium as a superconducting material for high-impedance quantum circuits, Nat. Mater. 18, 816 (2019).

[15] M. T. Bell, I. A. Sadovskyy, L. B. Ioffe, A. Y. Kitaev, and M. E. Gershenson, Quantum Superinductor with Tunable Nonlinearity, Phys. Rev. Lett. 109, 137003 (2012).

[16] A. Mizel and Y. Yanay, Right-sizing fluxonium against charge noise, Phys. Rev. B 102, 014512 (2020).

[17] A. Di Paolo, T. E. Baker, A. Foley, D. Sénéchal, and A. Blais, Efficient modeling of superconducting quantum circuits with tensor networks, npj Quantum Inf. 7, 11 (2021).

[18] K. Cedergren, R. Ackroyd, S. Kafanov, N. Vogt, A. Shnirman, and T. Duty, Insulating Josephson Junction Chains as Pinned Luttinger Liquids, Phys. Rev. Lett. 119, 167701 (2017).

[19] F. Piquemal and G. Genevés, Argument for a direct realization of the quantum metrological triangle, Metrologia 37, 207 (2000).

[20] J. B. Hertzberg, E. J. Zhang, S. Rosenblatt, E. Magesan, J. A. Smolin, J.-B. Yau, V. P. Adiga, M. Sandberg, M. Brink,
J. M. Chow, and J. S. Orcutt, Laser-annealing Josephson junctions for yielding scaled-up superconducting quantum processors, ArXiv:2009.00781 (2020).

[21] P. K. Day, H. G. LeDuc, B. A. Mazin, A. Vayonakis, and J. Zmuidzinas, A broadband superconducting detector suitable for use in large arrays, Nature 425, 817 (2003).

[22] M. V. Feigel'man and M. A. Skvortsov, Universal Broadening of the Bardeen-Cooper-Schrieffer Coherence Peak of Disordered Superconducting Films, Phys. Rev. Lett. 109, 147002 (2012).

[23] A. Bespalov, M. Houzet, J. S. Meyer, and Y. V. Nazarov, Theoretical Model to Explain Excess of Quasiparticles in Superconductors, Phys. Rev. Lett. 117, 117002 (2016).

[24] L. Grünhaupt, N. Maleeva, S. T. Skacel, M. Calvo, F. LevyBertrand, A. V. Ustinov, H. Rotzinger, A. Monfardini, G. Catelani, and I. M. Pop, Loss Mechanisms and Quasiparticle Dynamics in Superconducting Microwave Resonators Made of Thin-Film Granular Aluminum, Phys. Rev. Lett. 121, 117001 (2018).

[25] I. M. Pop, K. Geerlings, G. Catelani, R. J. Schoelkopf, L. I. Glazman, and M. H. Devoret, Coherent suppression of electromagnetic dissipation due to superconducting quasiparticles, Nature 508, 369 (2014).

[26] J. T. Peltonen, P. C. J. J. Coumou, Z. H. Peng, T. M. Klapwijk, J. S. Tsai, and O. V. Astafiev, Hybrid rf SQUID qubit based on high kinetic inductance, Sci. Rep. 8, 10033 (2018).

[27] T. M. Hazard, A. Gyenis, A. Di Paolo, A. T. Asfaw, S. A. Lyon, A. Blais, and A. A. Houck, Nanowire Superinductance Fluxonium Qubit, Phys. Rev. Lett. 122, 010504 (2019).

[28] H. Zhang, S. Chakram, T. Roy, N. Earnest, Y. Lu, Z. Huang, D. K. Weiss, J. Koch, and D. I. Schuster, Universal FastFlux Control of a Coherent, Low-Frequency Qubit, Phys. Rev. X 11, 011010 (2021).

[29] F. Yan, S. Gustavsson, A. Kamal, J. Birenbaum, A. P. Sears, D. Hover, T. J. Gudmundsen, D. Rosenberg, G. Samach, S. Weber, J. L. Yoder, T. P. Orlando, J. Clarke, A. J. Kerman, and W. D. Oliver, The flux qubit revisited to enhance coherence and reproducibility, Nat. Commun. 7, 12964 (2016).

[30] S. Gladchenko, D. Olaya, E. Dupont-Ferrier, B. Douçot, L. B. Ioffe, and M. E. Gershenson, Superconducting nanocircuits for topologically protected qubits, Nat. Phys. 5, 48 (2008).

[31] W. C. Smith, M. Villiers, A. Marquet, J. Palomo, M. R. Delbecq, T. Kontos, P. Campagne-Ibarcq, B. Douçot, and Z. Leghtas, Magnifying quantum phase fluctuations with Cooper-pair pairing, ArXiv:2010.15488 (2020).

[32] B. Douçot and L. B. Ioffe, Physical implementation of protected qubits, Rep. Prog. Phys. 75, 072001 (2012).

[33] M. T. Bell, J. Paramanandam, L. B. Ioffe, and M. E. Gershenson, Protected Josephson Rhombus Chains, Phys. Rev. Lett. 112, 167001 (2014).

[34] D. T. Le, A. Grimsmo, C. Müller, and T. M. Stace, Doubly nonlinear superconducting qubit, Phys. Rev. A 100, 062321 (2019).

[35] T. Larsen, M. Gershenson, L. Casparis, A. Kringhøj, N. Pearson, R. McNeil, F. Kuemmeth, P. Krogstrup, K. Petersson, and C. Marcus, Parity-Protected SuperconductorSemiconductor Qubit, Phys. Rev. Lett. 125, 056801 (2020). 
[36] A. Gyenis, P. S. Mundada, A. Di Paolo, T. M. Hazard, X. You, D. I. Schuster, J. Koch, A. Blais, and A. A. Houck, Experimental realization of a protected superconducting circuit derived from the $0-\pi$ qubit, PRX Quantum 2, 010339 (2021).

[37] P. Brooks, A. Kitaev, and J. Preskill, Protected gates for superconducting qubits, Phys. Rev. A 87, 052306 (2013).

[38] P. Groszkowski, A. D. Paolo, A. L. Grimsmo, A. Blais, D. I. Schuster, A. A. Houck, and J. Koch, Coherence properties of the $0-\pi$ qubit, New J. Phys. 20, 043053 (2018).

[39] M. T. Bell, W. Zhang, L. B. Ioffe, and M. E. Gershenson, Spectroscopic Evidence of the Aharonov-Casher Effect in a Cooper Pair Box, Phys. Rev. Lett. 116, 107002 (2016).

[40] K. Kalashnikov, W. T. Hsieh, W. Zhang, W.-S. Lu, P. Kamenov, A. D. Paolo, A. Blais, M. E. Gershenson, and M. Bell, Bifluxon: Fluxon-parity-protected superconducting qubit, PRX Quantum 1, 010307 (2020).

[41] W. C. Smith, A. Kou, X. Xiao, U. Vool, and M. H. Devoret, Superconducting circuit protected by two-Cooper-pair tunneling, npj Quantum Inf. 6, 8 (2020).

[42] R. J. Schoelkopf, A. A. Clerk, S. M. Girvin, K. W. Lehnert, and M. H. Devoret, in Quantum Noise in Mesoscopic Physics, edited by Y. V. Nazarov (Springer Netherlands, Dordrecht, 2003), p. 175.

[43] A. Grimm, N. E. Frattini, S. Puri, S. O. Mundhada, S. Touzard, M. Mirrahimi, S. M. Girvin, S. Shankar, and M. H. Devoret, Stabilization and operation of a Kerr-cat qubit, Nature 584, 205 (2020).

[44] U. Vool, A. Kou, W. Smith, N. Frattini, K. Serniak, P. Reinhold, I. Pop, S. Shankar, L. Frunzio, S. Girvin, and M. Devoret, Driving Forbidden Transitions in the Fluxonium Artificial Atom, Phys. Rev. Appl. 9, 054046 (2018).

[45] J. Koch, V. Manucharyan, M. H. Devoret, and L. I. Glazman, Charging Effects in the Inductively Shunted Josephson Junction, Phys. Rev. Lett. 103, 217004 (2009).

[46] J. Ulrich and F. Hassler, Dual approach to circuit quantization using loop charges, Phys. Rev. B 94, 094505 (2016).

[47] J. E. Mooij and Y. V. Nazarov, Superconducting nanowires as quantum phase-slip junctions, Nat. Phys. 2, 169 (2006).

[48] S. S. Mohan, M. del Mar Hershenson, S. P. Boyd, and T. H. Lee, Simple accurate expressions for planar spiral inductances, IEEE J. Solid-State Circuits 34, 1419 (1999).
[49] P. K. Mogensen and A. N. Riseth, OPTIM: A mathematical optimization package for JULIA, J. Open Source Softw. 3, 615 (2018).

[50] G. Szep and M. Peruzzo, Hamiltonian-optimization (2021). https://github.com/gszep/hamiltonian-optimisation.

[51] D. I. Schuster, A. A. Houck, J. A. Schreier, A. Wallraff, J. M. Gambetta, A. Blais, L. Frunzio, J. Majer, B. Johnson, M. H. Devoret, S. M. Girvin, and R. J. Schoelkopf, Resolving photon number states in a superconducting circuit, Nature 445, 515 (2007).

[52] P. Groszkowski and J. Koch, Scqubits: a Python package for superconducting qubits, arXiv:2107.08552 (2021).

[53] C. Rigetti, J. M. Gambetta, S. Poletto, B. L. T. Plourde, J. M. Chow, A. D. Córcoles, J. A. Smolin, S. T. Merkel, J. R. Rozen, G. A. Keefe, M. B. Rothwell, M. B. Ketchen, and M. Steffen, Superconducting qubit in a waveguide cavity with a coherence time approaching $0.1 \mathrm{~ms}$, Phys. Rev. B 86, 100506 (2012).

[54] J. Braümuller, L. Ding, A. P. Vepsäläinen, Y. Sung, M. Kjaergaard, T. Menke, R. Winik, D. Kim, B. M. Niedzielski, A. Melville, J. L. Yoder, C. F. Hirjibehedin, T. P. Orlando, S. Gustavsson, and W. D. Oliver, Characterizing and Optimizing Qubit Coherence Based on Squid Geometry, Phys. Rev. Appl. 13, 054079 (2020).

[55] S. Girvin, R.-S. Huang, A. Blais, A. Wallraff, and R. Schoelkopf, in Quantum Entanglement and Information Processing Ecole d'ete de Physique des Houches Session LXXIX, Les Houches, Vol. 79, edited by J.-M. R. Daniel Esteve and J. Dalibard (Elsevier, Les Houches, Haute-Savoie, Auvergne-Rhône-Alpes, France, 2004), p. 591.

[56] G. Wendin, Quantum information processing with superconducting circuits: A review, Rep. Prog. Phys. 80, 106001 (2017).

[57] R. Lescanne, L. Verney, Q. Ficheux, M. H. Devoret, B. Huard, M. Mirrahimi, and Z. Leghtas, Escape of a Driven Quantum Josephson Circuit into Unconfined States, Phys. Rev. Appl. 11, 014030 (2019).

[58] Matilda Peruzzo, Farid Hassani, Gregory Szep, Andrea Trioni, Elena Redchenko, Martin Žemlička, and Johannes M. Fink, Data for: "Geometric Superinductance Qubits: Controlling Phase Delocalization across a Single Josephson Junction," https://doi.org/10.5281/zenodo.5592104 (2021). 